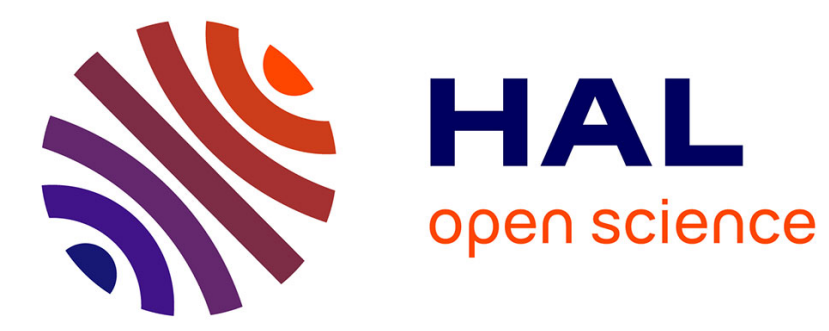

\title{
User Selection in 5G Heterogeneous Networks Based on Millimeter-Wave and Beamforming
}

\author{
Ahmad Fadel, Bernard Cousin, Ayman Khalil
}

\section{To cite this version:}

Ahmad Fadel, Bernard Cousin, Ayman Khalil. User Selection in 5G Heterogeneous Networks Based on Millimeter-Wave and Beamforming. IEEE HPCC Conference, Jun 2018, Exeter, United Kingdom. 10.1109/HPCC/SmartCity/DSS.2018.00097 . hal-01798960

\section{HAL Id: hal-01798960 \\ https://hal.science/hal-01798960}

Submitted on 24 May 2018

HAL is a multi-disciplinary open access archive for the deposit and dissemination of scientific research documents, whether they are published or not. The documents may come from teaching and research institutions in France or abroad, or from public or private research centers.
L'archive ouverte pluridisciplinaire HAL, est destinée au dépôt et à la diffusion de documents scientifiques de niveau recherche, publiés ou non, émanant des établissements d'enseignement et de recherche français ou étrangers, des laboratoires publics ou privés. 


\section{User Selection in 5G Heterogeneous Networks Based on Millimeter-Wave and Beamforming}

\author{
Ahmad FADEL \\ IRISA \\ University of Rennes 1 \\ Rennes, France \\ ahmad.fadel(at)irisa.fr
}

\author{
Bernard COUSIN \\ IRISA \\ University of Rennes 1 \\ Rennes, France
}

\author{
Ayman KHALIL \\ IUL \\ CCE Department \\ Wardanieh, Lebanon
}

\begin{abstract}
Heterogeneous Networks (HetNETs) are considered as an effective solution to improve the coverage and system throughput for future cellular networks. The extremely growing mobile market, together with the arising demand for high data rates, motivate us to open a new spectrum related to millimeter waves (mmwaves) while using beamforming that can serve simultaneously a group of users. In this paper, we formulate an optimization problem for HetNETs multi-user selection in a multi-input-multi-output and orthogonal frequency-division multiple access (MIMO-OFDMA) system, aiming to maximize the total system throughput. We solve the problem by applying a modified version of well-known metaheuristic algorithms. The optimal solution is obtained using an exhaustive search algorithm that provides an ideal solution which is complex to be computed. Greedy zero-forcing dirty-paper gZF-DP and zeroforcing selection ZFS algorithms were selected from literature for the sub-optimal solution. In parallel, a water-filling algorithm has been optimized after adding new power constraint and it has been used for power distribution. Hence, we analyze the throughput performance of our systems using throughput metric. The results show that ZFS outperforms gZF-DP algorithm as it achieves higher total throughput, While gZF-DP outperforms ZFS algorithm in the execution time.
\end{abstract}

Index Terms-User selection, 5G heterogeneous network, beamforming, milli-meter waves.

\section{INTRODUCTION}

$5 \mathrm{G}$ the next generation of the wireless communication system will be able to handle a thousand times more traffic than today's networks, and it will be up to 10 times faster than $4 \mathrm{G}$ LTE. There are five new technologies emerging as a foundation of 5G. Millimeter waves (mmwaves), small cells, massive multi-input-multi-output MIMO, beamforming and full duplex [1].

Mmwaves gives us the opportunity to use a new spectrum of band, that fall between 30 and $300 \mathrm{GHz}$ [2]. This section of the spectrum has never been used before for mobile devices and opening it up means more bandwidth for everyone, especially after having all frequency spectrum bands under $6 \mathrm{GHz}$ started to get more crowded. But there is a drawback millimeter wave communications suffer from huge propagation loss compared with other communication systems in using lower carrier frequencies. Accordingly, this fact limits their range to approximately $200 \mathrm{~m}$. Therefore to figure out this problem the concept of heterogeneous networks HetNETs has been introduced while densifying the macrocell by small cells. Doubtless, HetNETS is a promising solution for the capacity enhancement in the 5G cellular networks [2]. Under those circumstances, small cells are appropriate for the utilization of mmwaves.

Proportionally, high frequency means small antenna size. This can be seen as a good achievement to have massive MIMO technology, where hundred of antenna elements can be integrated easily. Thus, increasing the throughput of today's networks by the factor $N_{t} \mathrm{x} N_{r}$ [3], where $N_{t}$ and $N_{r}$ are the number of transmitted and received antenna respectively. Broadcasting information in every direction at once could cause serious interference problem which brings us to the use of beamforming techniques. Beamforming is like a traffic signaling system for cellular signals, so it allows to send a focused stream to a specific direction hence this precision prevent interference [4].

Combining all $5 \mathrm{G}$ technologies in one system is required and must be studied. Indeed, orthogonal frequency division multiple access (OFDMA) will be considered as a promising broadband cellular platform to support high-speed wireless communication [1], and it's the unquestionable front-runner for 5G. Moreover, we have a recent interest in transmit beamforming strategies for the cellular downlink that aim to attain the total throughput of the wireless channel.

k-tier heterogeneous downlink Cellular Networks proposed in the literature [5] focused on cooperation between different tiers. The authors in [6] have formulated an optimization problem for joint beamforming, resource allocation and scheduling for multi-cell multi-user selection, that achieve a total average weighted system throughput maximization. Investigate the design and the associated tradeoffs between energy efficiency and system throughput maximization in a Twotier heterogeneous network. This is obtained by introducing active/sleep (on/off) modes in macrocell base station [7], [8]. A reduced-complexity suboptimal solution to total throughput maximization is suggested in [9]. It proposes the use of QR decomposition of the channel combined with dirty-paper DP coding at the transmitter. In that case, this combination of both approaches, nulls interference between data streams, and hence, it is called zero-forcing dirty-paper (ZF-DP) precoding [9]. Zero forcing algorithms ZFS was applied in a single 
cell multi-user selection while using statistical channel model where path loss is normalized and the study is based on the 3rd technology of wireless communication networks [10].

Our goal is to investigate low-complexity downlink beamforming solution for the proposed optimization problem to meet the overwhelming demands of network throughput for a practically important case wherein the number of users $N_{u}$ is larger than the number of transmit antennas $N_{t}$, which entails user selection. Our aim is four-fold: i) Formulate an optimization problem for joint beamforming, power allocation, and user selection, in a heterogeneous cellular network based on two different technologies $(4 \mathrm{G})$ and $(5 \mathrm{G})$, for the purpose of maximizing the system throughput; ii) compute the optimal solution by applying the ES algorithm that will be considered as the maximum threshold for all other solutions; iii) propose an alternative modified version for both gZF-DP and ZFS, to be adaptable for the suggested channel model; iv) assess the performance of both gZF-DP and ZFS compared to that of ES, while total throughput is considered as the evaluation metric.

The residual of the paper is organized as follows: Section II outlines the system and channel models. In Section III we formulate the joint beamforming, resource allocation, and scheduling optimization problem [6]. Section IV describes the optimal and reduced-complexity algorithms. Furthermore, Section $\mathrm{V}$ provides a simulation-based comparison of the system throughput performance for ES, gZF-DP and ZFS algorithms. Conclusions and future work are mentioned in Section VI.

\section{HeTNET MULTI USER MIMO-OFDMA NETWORK MODEL}

\section{A. HetNet MULTI USER MIMO-OFDMA System Model}

A heterogeneous multi-user MIMO-OFDMA network is considered, consisting of $M$ macrocells densified by $P$ picocells with a $K$ user equipment (UEs) distributed overall the network as depicted in Figure 1. Each base station (BS) is equipped with $N_{t}$ transmit antennas, and each UE with $N_{r}$ receive antennas.

Spatial multiplexing is the most attracted characteristic in MIMO systems because each BS can serve up to $\frac{N_{t}}{N_{r}}$ UEs simultaneously for each resource unit. Frequencies are attributed as follows: we associate for macrocell the $4 \mathrm{G}$ frequency spectrum, and for picocells mmwaves that fall in the spectrum range of $5 \mathrm{G}$. Thanks to fiber optic backhaul links, macro and pico BSs are connected to a centralized control unit. We assume that the wireless channel operates in time-divisionmultiplexing (TDD) mode to provide reciprocity, by which the uplink channel is used as an estimate of the downlink channel, and this occurs when receiving a pilot training sequence from terminal devices toward the base station where channel state information (CSI) estimation takes place.

\section{B. Channel Model}

An OFDMA system is considered with $T$ resource units with $h_{n k}(t) \in \mathcal{C}^{N_{t} \times N_{r}}$ to be the channel vector between the $(m, p)$ th BS and the $k$ th UE of the $\left(m^{\prime}, p^{\prime}\right)$ th cell in the $t$ th

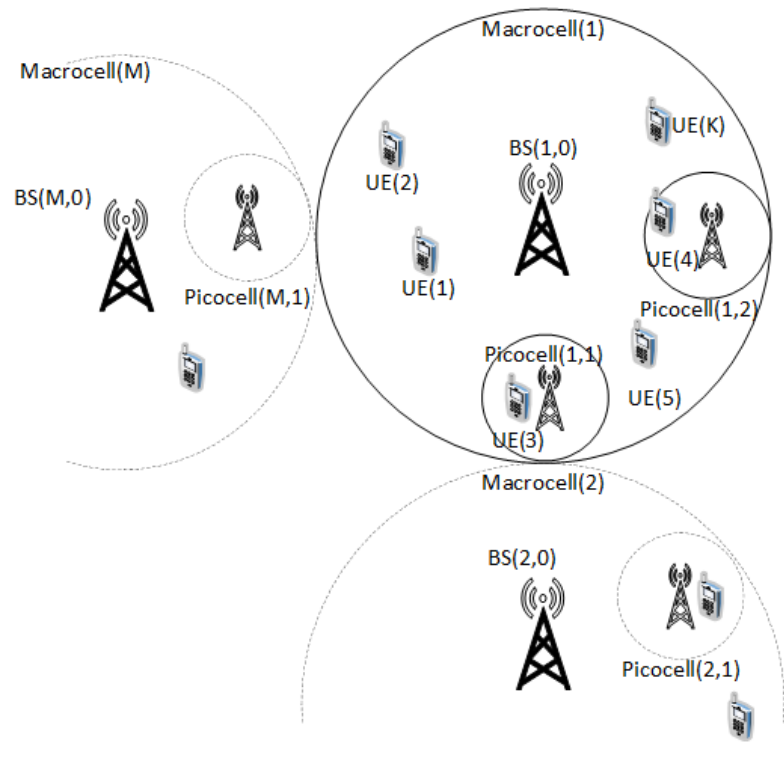

Figure 1. Heterogeneous network with MIMO base stations.

resource unit. Where $m$ and $p$ are the indices of the distributed cells in the network, knowing that $m \in[1 \ldots M]$ and $p \in$ $[0 \ldots P]$, for clarification $p=0$ identified the $\mathrm{BS}$ macrocell. Then, the received signal at the $k t h \mathrm{UE}$ of the $\left(m^{\prime}, p^{\prime}\right)$ th cell in the $t$ th resource unit, $y_{n k(t)}$, is given by

$$
\begin{aligned}
& y_{n k(t)}=S_{n k}(t) h_{n k}(t) w_{n k}(t) x_{n k}(t)+I_{n k}(t)+n_{u}, \\
& \forall n=(m, p) \text { with } m \in[1 \ldots M] \text { and } p \in[0 \ldots P]
\end{aligned}
$$

where $S_{n k}(t) \in\{0,1\}$ denotes the resource unit allocation indicator, $w_{n k}(t)$ and $x_{n k}(t)$ are the transmit power-scaled beamforming and the transmit symbol for the link between the $(m, p)$ th $\mathrm{BS}$ and the $k$ th $\mathrm{UE}$ in the $t$ th resource unit, respectively. Furthermore, $h_{n k}(t)=l_{n k}(t) g_{n k}(t) \tilde{h}_{n k}(t)$, where $l_{n k}(t), g_{n k}(t)$, represent respectively the path loss, shadowing, where they are generated using rapapport model [11], and what is worth to be mentioned is that this model is realistic. Then, $\tilde{h}_{n k}(t)$ small scale fading coefficients are generated using [12] that provides a statistical channel model. In addition, $n_{u} \sim \mathcal{N}\left(\mathbf{0}, \sigma^{2} I_{n k}(t)\right)$ is the additive white Gaussian noise (AWGN) corrupting the received signal with zero mean and variance $\sigma^{2}$ and $I_{n k}(t)$ is the interference.

\section{Problem Formulation}

In [6], the authors have formulated an optimization problem for a multi-cell network, assuming universal frequency reuse. We reformulate this optimization problem to be compatible with the proposed system model, where we are dealing with HetNets using mmwaves only for picocells.

\section{A. System Performance Evaluation Metric}

The system throughput will be used as a metric unit to evaluate the proposed system. The instantaneous channel throughput for the $k$ th user of the $n$th cell in the $t$ th resource unit is given by 


$$
\begin{aligned}
& R_{n k}(t)=B_{w} \log _{2}\left(1+\zeta_{n k}(t)\right), \\
& \forall n=(m, p) \text { with } m \in[1 \ldots M] \text { and } p \in[0 \ldots P]
\end{aligned}
$$

where $B_{w}$ is the channel bandwidth and $\zeta$ denotes the received signal-to-interference-plus-noise-ratio (SINR) given by

$$
\zeta_{n k}(t)=\frac{\left|h_{n k}(t) w_{n k}(t)\right|^{2}}{I_{n k}(t)+\sigma^{2}}, \quad \forall n=(m, p)
$$

where $I_{n k}(t)$ represents the received intracell multi-user interference. While picocells are placed far enough from each other to avoid intercell interference.

The performance evaluation metric for the proposed problem formulation is the average system throughput, which is defined by $\mathbb{U}$ as the total average number of bit/s/Hz/BS successfully received at the $k$ th UEs, worth mentioning that we are dealing with HetNets on the contrary to [6]. Thus respecting the different characteristics of each type of cell (macro, pico) is unquestionable, like the size and different technologies adopted in each cell. The corresponding expression is given by

$$
\begin{array}{r}
\mathbb{U}(\mathbb{B}, \mathbb{P}, \mathbb{S})=\frac{1}{M *(P+1)} \sum_{m=1}^{M} \sum_{p=0}^{P} \sum_{k \in U_{n}} \sum_{t=1}^{T} R_{n k}(t) \\
\forall n=(m, p) \text { with } m \in[1 \ldots M] \text { and } p \in[0 \ldots P]
\end{array}
$$

where $\mathbb{B}, \mathbb{P}$ and $\mathbb{S}$, refer to the beamforming, power allocation and scheduling policies respectively. $U_{n}, \forall n=(m, p)$, denotes the set of UEs served by each macro and pico BSs. $P$ and $M$ are the total number of picocells and macrocells in the network respectively.

\section{B. Formulation of the Optmization Problem}

In this subsection, a new optimization problem $(\mathrm{P})$ is formulated as follows:

$$
\left\{\begin{array}{l}
(\mathbb{B}, \mathbb{P}, \mathbb{S})^{*}=\arg \max \mathbb{U}(\mathbb{B}, \mathbb{P}, \mathbb{S}) \\
\text { s.t, } C 1: S_{n k}(t) \in\{0,1\}, \forall n=(m, p), k, t \\
C 2: \sum_{k \in U_{n}} S_{n k}(t) \leq \frac{N t}{N r}, \forall n=(m, p), t \\
C 3: \sum_{k \in U_{n}} \sum_{t=1}^{T} S_{n k}(t)\left\|w_{n k(t)}\right\|^{2} \leq P_{n}, \forall n=(m, p) \\
C 4: \sum_{n} \sum_{t=1}^{T} S_{n k}(t) \leq 1, \quad \forall k, n=(m, p) \\
\text { with } m \in[1 \ldots M] \text { and } p \in[0 \ldots P]
\end{array}\right.
$$

where $(\mathbb{B}, \mathbb{P}, \mathbb{S})^{*}$ refers to the optimal beamforming power allocation, and scheduling policies respectively. $\mathrm{C} 1$ indicates whether the resource unit is allocated to the UE or not, in each macro or picocells. C2 guarantees that a resource unit is only assigned to all most $\frac{N t}{N r}$ UEs in one resource unit, again in each macro or picocells. C3 guarantees that the individual transmit power constraint for each BS [6] will not exceed a certain maximum $P_{n}$. C4 assures that users could take maximum one and only one resource unit during each selection.

Solving this optimization problem analytically presents a high complexity, thus we propose to use metaheuristic algorithms to find a sub-optimal solution that can give us an overview of the first result in the proposed system. For the rest of this paper, we will consider one resource unit for each BS to be shared between users, hence this means that $t=1$ for each BS. The reason from this assumption is to simplify the simulation part.

\section{OPTIMAL AND LOW-COMPLEXITY ALGORITHMS}

In this section, we will apply three algorithms ES, gZFDP and gZF that take into consideration the same constraints and objective of the optimization problem (P). Accordingly, the distributed power between users should be less or equal the maximum power in each BS. The number of users that share the same resource unit should not exceed the number of antenna elements installed on a BS.

\section{A. Exhausitve Search Algorithm}

Maximizing the system throughput is the most important target of telecommunication operators in the next generation of cellular networks. Thus, high throughput enables to reply quickly to users, thus it achieves their satisfaction.

The objective of the optimization problem $(\mathrm{P})$, is selecting $r \leq N_{t}$ users and respecting all the aforementioned constraint in a way to maximize the system throughput, while using beamforming and best power allocation. To achieve the goal, we suggest getting the optimal solution first, through applying the exhaustive search algorithm. This algorithm checks all the possible combinations in a way to get the optimal solution by selecting a set of users that achieve the maximum total throughput.

In Algorithm 1, we propose to select $r \leq N_{t}$ from $N_{u}$ users, by trying all the possible combinations to achieve the maximum total throughput. Power is uniformly distributed among the selected users.

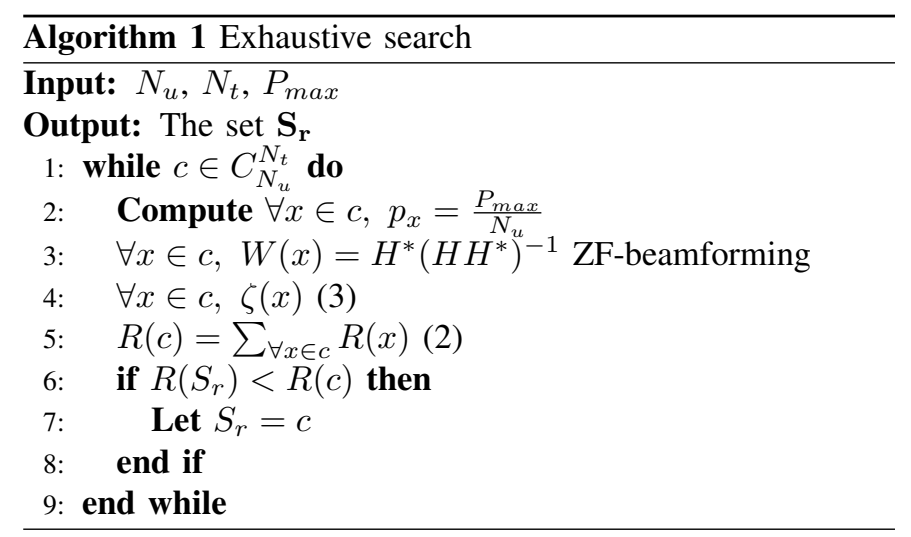

Consider $h_{n k} \forall n=(m, p)$ where $m \in[1 \ldots M]$ and $p \in$ $[0 \ldots P]$ the channel vector between the $N_{t}$ transmit antenna 
elements and the received antenna of $k$ users in each macrocell and picocells for all algorithms.

We denote $\mathbf{h}_{\mathbf{k}}:=\left[\begin{array}{lllll}\mathbf{h}_{\mathbf{k}, \mathbf{1}} & \mathbf{h}_{\mathbf{k}, \mathbf{2}} & \ldots & \mathbf{h}_{\mathbf{k}, \mathbf{N}_{\mathrm{t}}}\end{array}\right]$ a row vector. Thus, the channel matrix $\mathbf{H}$ is

$$
\mathbf{H}=\left[\begin{array}{llll}
\mathbf{h}_{\mathbf{1}}^{*} & \mathbf{h}_{\mathbf{2}}^{*} & \ldots & \mathbf{h}_{\mathbf{k}}^{*}
\end{array}\right]^{*}
$$

Where $(.)^{*}$ is the conjugate-transpose, and the received signal at user $k$, and $W$ represents a specific type of beamforming which is ZF. Let $\mathbf{w}_{\mathbf{k}}:=\left[\begin{array}{lllll}\mathbf{w}_{\mathbf{k}, \mathbf{1}} & \mathbf{w}_{\mathbf{k}, \mathbf{2}} & \ldots & \mathbf{w}_{\mathbf{k}, \mathbf{N}_{\mathbf{t}}}\end{array}\right]^{\mathbf{T}}\left((.)^{T}\right.$ denotes transpose) be the beamforming weight vector for user $k$. The beamforming weight matrix $\mathbf{W}$ is

$$
\mathbf{W}=\left[\begin{array}{llll}
\mathbf{w}_{1} & \mathbf{w}_{2} & \ldots & \mathbf{w}_{\mathbf{k}}
\end{array}\right]
$$

The complexity of getting this optimal solution motivate us to find a less complex algorithm. The greedy algorithms get a sub-optimal solution that makes a trade-off for the computing time. This is what will be presented in the upcoming proposed algorithms.

\section{B. Greedy Zero-Forcing Dirty-Paper Algorithm}

The authors in [9] have proposed a suboptimal solution to maximize (3), based on the QR-type decomposition [13] of the channel $\mathbf{H}=\mathbf{L Q}$ matrix obtained by applying Gram-Schmidt orthogonalization to the rows of $\mathbf{H}$, while computing $P_{r}^{\perp}$ the projector matrix. $\mathbf{L}$ is a lower triangular matrix, and has orthonormal rows. Setting $W=Q^{*}$. They have proposed a multiplexing technique based on coding for known interference, known as "Writing on Dirty Paper," Costa precoding [14], or dirty paper (DP) coding. The combination of both approaches QR-decomposition and DP coding at the transmitter, nulls interference between data stream. Authors of [9] have applied the quite same greedy solution, on a single cell for $3 \mathrm{G}$ technology, as they considered a statistical channel model with normalized pathloss which is not the case in our proposed system model since we are using the mmwave channel model of Rappaport [11] for picocells and Friis model [15] for macrocell.

Power is allocated using the water-filling algorithm, whereas it allocates the power among users taking into account the channel radio condition, where it distributes high power for users that have good radio condition and low power if not, see Appendix A. Indeed, using this algorithm is more effective than distributing the power equally between users, since attributing a high power for bad radio condition is useless. We have formulated a water-filling optimization problem, adding a new constraint that guarantees a minimum power $\alpha_{i}$ for all selected users. $\lambda$ is the solution of the water-filling equation and $g_{i}$ is the radio condition.

$$
\sum_{i=1}^{K}\left[\lambda-\frac{1}{g_{i}}\right]=P_{\max }
$$

$$
\begin{gathered}
p_{i}=\left[\lambda-\frac{1}{g_{i}}\right] \\
\text { if } p_{i}<\alpha_{i} \text { then } p_{i}=\alpha_{i}
\end{gathered}
$$

The algorithm 2 is called gZF-DP because it may produce a local maximum solution and not the optimal one. Let $U_{n}=$ $\{1,2, \ldots, K\}$ be the set of indices of all UEs, and $\mathrm{Sr}=$ $\left\{s_{1}, \ldots, s_{r}\right\} \subset U_{r}$.

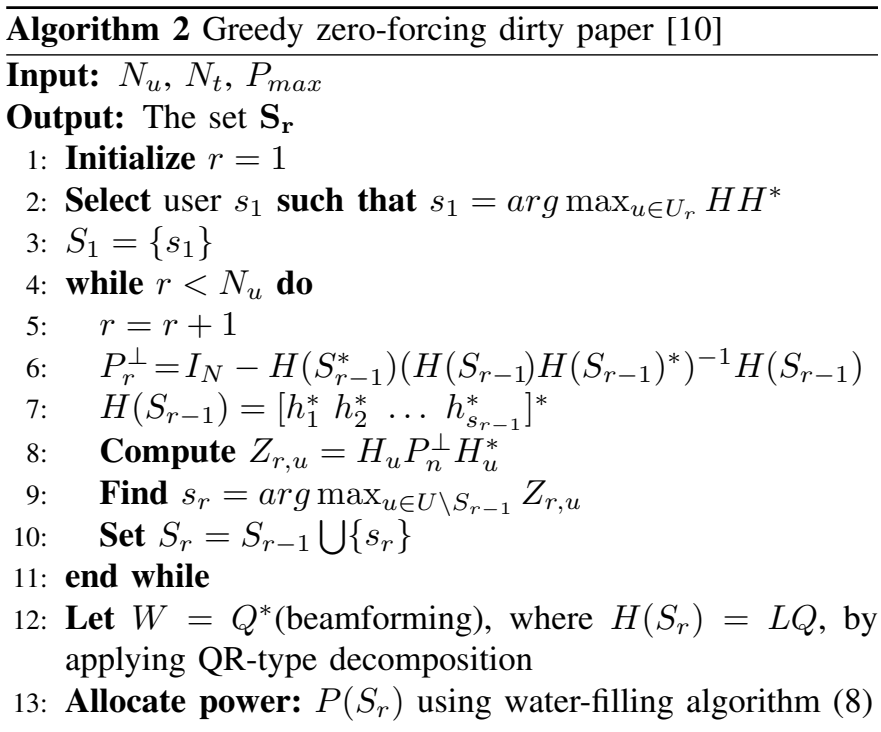

\section{Greedy Zero-Forcing Algorithm}

$\mathrm{ZF}$ beamforming is a method of spatial signal processing by which the multiple antenna transmitters can null multiuser interference signals in wireless communications. This occurs by inverting the channel matrix at the transmitter so that orthogonal channels between transmitter and receivers are created. This problem is solved by ES, which maximizes $R\left(S_{r}\right)$ the total throughput of the set $S_{r}$. However such algorithm has prohibitive complexity. Again power is distributed among users using water-filling (8). We have modified the algorithm in [10], at the beginning SNR is calculated for the first selected user since it does not experience any kind of interference. Next users are selected after calculating their SINR, in a way to achieve a maximum throughput. Algorithm 3 does not use dirty paper DP coding, as orthogonality is not the only criterion for selecting users, hence it selects the user that provides higher throughput.

\section{EVALUATION AND PERFORMANCE ANALYSIS}

In this section, we evaluate the performance of gZF-DP and that of ZFS. Then we compare both algorithms with the optimal one, the exhaustive search, via Monte-Carlos simulations. Hence we average the total throughput over 1,000 random channel realizations. All BSs are assumed to transmit with identical power when using the exhaustive algorithm. Water-filling is adopted for power allocation in both gZF-DP 

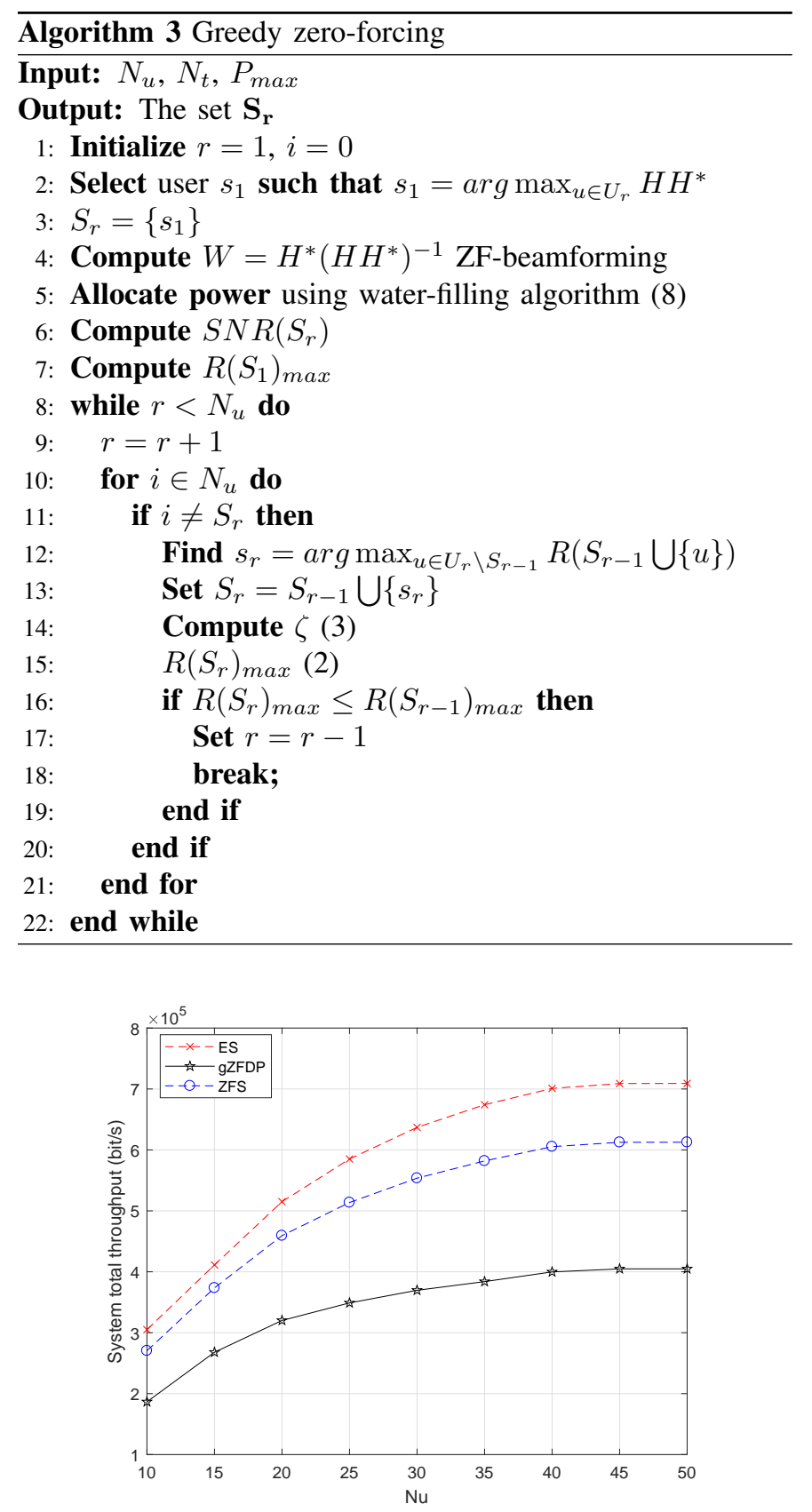

Figure 2. Total throughput for macrocells versus $N_{u}$ users.

and that of ZFS. The simulation parameters are given in Table II.

\section{A. System Throughput versus number of Users in Macro Cells}

Figure 2 depicts the total system throughput for both ZFS and gZF-DP to be compared with the optimal solution ES for the macrocells. As shown the three curves are dramatically increasing to reach a maximum threshold. We can detect that ZFS outperforms the gZF-DP, as it is close to the optimal solution. In Table I we have shown the relative error ZFS, gZF-DP and ES respectively. And after analyzing the result and referring to the calculated values, 0.118 is an acceptable

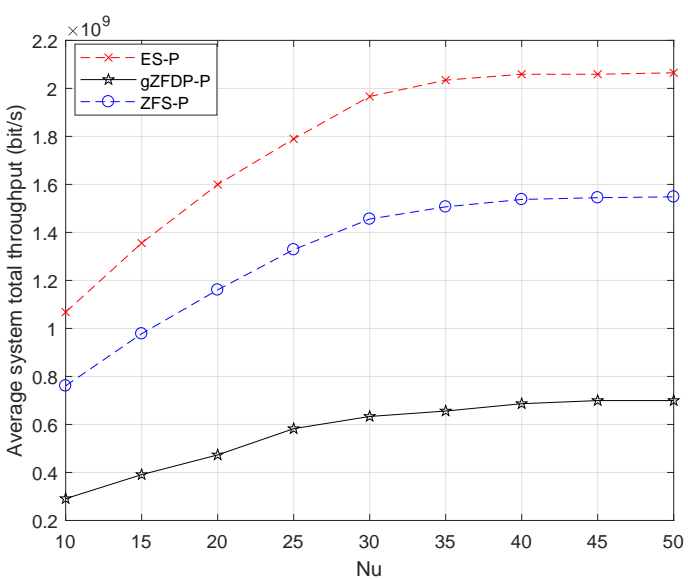

Figure 3. Average total throughput for picocells versus $N_{u}$ users.

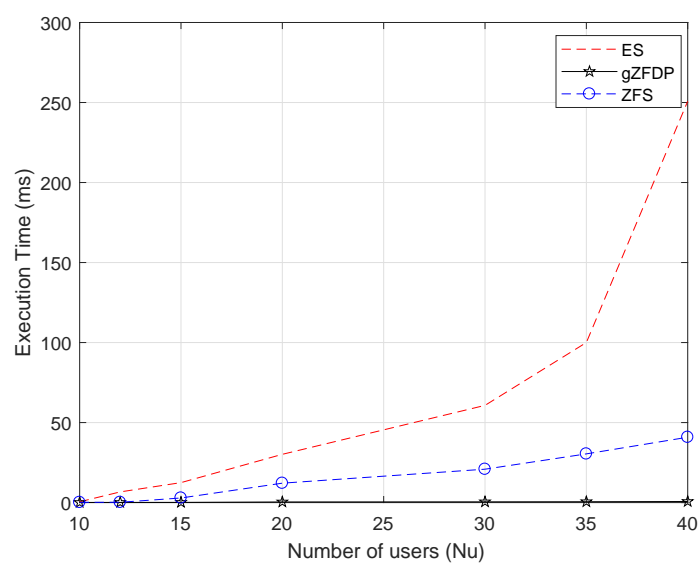

Figure 4. Execution time versus $N_{u}$ users

error compared to ES, especially taking into account that we would avoid the complexity of the exhaustive search.

\section{B. Average System Throughput versus number of Users in Pico cells}

In Figure 3 we show the average system throughput for both ZFS and gZF-DP to be compared with the optimal solution ES for the picocells. As shown the three curves are dramatically increasing to reach a saturated maximum threshold. We can clearly observe that ZFS outperforms the gZF-DP, as it is close to the optimal solution. In Table I we have shown the relative error ZFS with ES. Referring to the calculated values, 0.596 is an acceptable error compared to ES, especially if we take into account that we would avoid the complexity of the exhaustive search.

\section{System Execution Time}

Execution time is an important factor to be taken into consideration. Regarding Figure 4, execution time gZF-DP does not exceed $1 \mathrm{~ms}$ when $N_{u}=40$ users, while ZFS need $40.8421 \mathrm{~ms}$ for the same number of users. But regarding 
the ES algorithm, its curve increases exponentially and tends toward infinity as the number of users increases. To interpret this result, we can see that gZF-DP outperforms ZFS and ES using the metric of time, but ZFS gives an acceptable throughput compared to optimal thus it could be considered as a trade-off algorithm between gZF-DP and ES.

$$
\begin{aligned}
& \text { relative error }=\frac{E S-X}{E S} \\
& \forall X \in\{Z F S, g Z F-D P\}
\end{aligned}
$$

Table I

RELATIVE ERROR (10)BETWEEN BOTH ALGORITHMS AND ES

\begin{tabular}{|c|c|c|c|}
\hline relative & \multicolumn{3}{|c|}{ ZFS, gZF-DP versus ES } \\
\cline { 2 - 4 } error & $\boldsymbol{Z F S}-\boldsymbol{P}$ & $\boldsymbol{Z F S}$ & $\mathbf{g Z F}-\boldsymbol{D P}$ \\
\hline$N_{u}=30$ & 0.596 & 0.118 & 0.397 \\
\hline
\end{tabular}

Table II

SIMULATION PARAMETER

\begin{tabular}{|c|l|}
\hline Parameter & Value \\
\hline Channel model & Statistical channel [12] \\
\hline Center frequency $f_{c}$ & $\begin{array}{l}\text { Macro BS: } 2.6 \mathrm{GHz} \text {, pico BS: } 28 \\
\text { GHz }\end{array}$ \\
\hline Signal bandwidth & $\begin{array}{l}\text { Macro BS: } 180 \mathrm{kHz} \text { per resource } \\
\text { unit, pico BS: } 800 \mathrm{MHz}\end{array}$ \\
\hline Inter-site distance (ISD) for macro & $500 \mathrm{~m}$ \\
\hline Number of macro cells & 1 \\
\hline Number of pico cells & 2 \\
\hline$N_{t}:$ spacing & $\frac{\lambda}{2}$ \\
\hline Number of transmit \& receive antenna & $N_{t}=4, N_{r}=1$ \\
\hline Transmit power & $\begin{array}{l}\text { Macro BS: } 46 \mathrm{dBm}, \text { pico BS: } 26 \\
\text { dBm }\end{array}$ \\
\hline Noise power $\sigma$ & $\sigma=-174+10 \log B_{w} d B$ \\
\hline UE distribution & $\begin{array}{l}N_{u} \text { UEs uniformly distributed in } \\
\text { all system }\end{array}$ \\
\hline CSI at transmitter & perfect knowledge \\
\hline
\end{tabular}

\section{CONCLUSION}

In this paper we have formulate an optimization problem aiming to total throughput maximization for downlink OFDMA-MIMO system, using beamforming techniques and $5 \mathrm{G}$ HetNETs. We have presented our system model where we used a realistic channel model for mmwaves $5 \mathrm{G}$. We have selected two different metaheuristic algorithms from literature and we have modified them to be more efficient taking into account beamforming and mmwaves, as we have applied the exhaustive search algorithm to get the optimal solution. Numerical results revealed that ZFS algorithm outperforms gZF-DP as it provides higher system throughput and close to the optimal solution. But gZF-DP outperforms ZFS where it needs less execution time.

\section{APPENDIX A}

\section{A. Water filling}

Formulation of water filling algorithm that guarantees for users that suffer from a bad radio condition a minimum power $\alpha_{i}$ to send their data streams. This could be considered as ensuring fairness between the users while replying on their requests. The optimization problem is formulated as follows:

$$
\text { (P) }\left\{\begin{array}{l}
\max \quad \sum_{i}^{n} \log _{2}\left(1+g_{i} p_{i}\right) \\
\text { s.t } \quad \sum_{i=1}^{n} p_{i}=P_{\max } \\
\text { where } \quad \alpha_{i}=1 / g_{i}\left(2^{C_{\min }}-1\right) \\
p_{i} \geq \alpha_{i}
\end{array}\right.
$$

where $g_{i}$ and $p_{i}$ are the radio condition and power respectively for user i. Whereas $C_{\min }$ is a minimum capacity that user must achieve in the worst case.

$$
\frac{\partial \varphi}{\partial P}=\frac{g_{i}}{\ln 2\left(1+p_{i} g_{i}\right)}-\lambda=0
$$

Knowing that $\lambda$ refers to the water level, similarly, it is the solution of the water-filling.

$$
\lambda \ln 2=\frac{g_{i}}{1+g_{i} p_{i}} \Leftrightarrow \frac{g_{i}}{\lambda \ln 2}-1=p_{i} g_{i}
$$

Thus,

$$
\frac{1}{\lambda \ln 2}-\frac{1}{g_{i}}=p_{i}
$$

then,

$$
\begin{gathered}
\sum_{i=1}^{n} p_{i}=P_{\max } \\
\frac{n}{\lambda \ln 2}-\sum_{i=1}^{n} \frac{1}{g_{i}}=P_{\max } \\
\frac{1}{\lambda \ln 2}=\frac{P_{\max }+\sum_{i=1}^{n} \frac{1}{g_{i}}}{n} \\
p_{i}=\frac{P_{\max }+\sum_{i=1}^{n} \frac{1}{g_{i}}}{n}-\frac{1}{g_{i}}
\end{gathered}
$$

Particular condition:

$$
\text { if } p_{i}<\alpha_{i} \quad \text { then } \quad p_{i}=\alpha_{i}
$$

This algorithm is necessary especially while dealing with mmwaves technologies. Because channel model of mmwaves experience high pathloss which affect the radio condition where the user with bad channel does not get power where $p_{i}=0$, thus to avoid this case and respecting the constraint of the optimization problem, in worst case $p_{i}=\alpha_{i}$. 


\section{REFERENCES}

[1] J. G. Andrews, S. Buzzi, W. Choi, S. V. Hanly, A. Lozano, A. C. Soong, and J. C. Zhang, "What will 5g be?" IEEE Journal on selected areas in communications, vol. 32, no. 6, pp. 1065-1082, 2014.

[2] Y. Niu, Y. Li, D. Jin, L. Su, and A. V. Vasilakos, "A survey of millimeter wave (mmwave) communications for $5 \mathrm{~g}$ : opportunities and challenges. arxiv preprint," Wireless Networks, vol. 21, pp. 2657-2676, 2015.

[3] R. C. de Lamare, "Massive mimo systems: signal processing challenges and future trends," URSI Radio Science Bulletin, vol. 86, no. 4, pp. 8-20, 2013.

[4] S. Kutty and D. Sen, "Beamforming for millimeter wave communications: An inclusive survey," IEEE Communications Surveys \& Tutorials, vol. 18, no. 2, pp. 949-973, 2016.

[5] J. Park, N. Lee, and R. W. Heath Jr, "Feedback design for multiantenna k-tier heterogeneous downlink cellular networks," arXiv preprint arXiv:1708.03861, 2017.

[6] J. Zhu, R. Schober, and V. Bhargava, "Joint beamforming, resource allocation, and scheduling for multi-cell multi-user mimo-ofdma systems," in 2012 IEEE Global Communications Conference (GLOBECOM). IEEE, 2012, pp. 4594-4599.

[7] T. Q. Quek, W. C. Cheung, and M. Kountouris, "Energy efficiency analysis of two-tier heterogeneous networks," in 11th European Wireless Conference 2011-Sustainable Wireless Technologies (European Wireless). VDE, 2011, pp. 1-5.

[8] Y. S. Soh, T. Q. Quek, M. Kountouris, and H. Shin, "Energy efficient heterogeneous cellular networks," IEEE Journal on Selected Areas in Communications, vol. 31, no. 5, pp. 840-850, 2013.

[9] G. Caire and S. Shamai, "On the achievable throughput of a multiantenna gaussian broadcast channel," IEEE Transactions on Information Theory, vol. 49, no. 7, pp. 1691-1706, 2003.

[10] G. Dimic and N. D. Sidiropoulos, "On downlink beamforming with greedy user selection: performance analysis and a simple new algorithm," IEEE Transactions on Signal processing, vol. 53, no. 10, pp. $3857-3868,2005$

[11] M. R. Akdeniz, Y. Liu, M. K. Samimi, S. Sun, S. Rangan, T. S. Rappaport, and E. Erkip, "Millimeter wave channel modeling and cellular capacity evaluation," IEEE journal on selected areas in communications, vol. 32, no. 6, pp. 1164-1179, 2014.

[12] A. Alkhateeb, G. Leus, and R. W. Heath, "Limited feedback hybrid precoding for multi-user millimeter wave systems," IEEE transactions on wireless communications, vol. 14, no. 11, pp. 6481-6494, 2015.

[13] R. A. Horn and C. R. Johnson, Matrix analysis. Cambridge university press, 1990.

[14] M. Costa, "Writing on dirty paper (corresp.)," IEEE transactions on information theory, vol. 29, no. 3, pp. 439-441, 1983.

[15] F. Lassabe, P. Canalda, P. Chatonnay, F. Spies, and O. Baala, "A friisbased calibrated model for wifi terminals positioning," in Sixth IEEE International Symposium on a World of Wireless Mobile and Multimedia Networks, 2005. IEEE, 2005, pp. 382-387. 